\title{
Proteoglycans of human articular cartilage
}

\section{Identification of several populations of large and small proteoglycans and of hyaluronic acid-binding proteins in successive cartilage extracts}

\author{
Vladimir VILIM and Jana KRAJICKOVA \\ Research Institute of Rheumatology, Na slupi 4, 12850 Praha 2, Czechoslovakia
}

\begin{abstract}
Two specimens of human articular cartilage were successively extracted with solutions of phosphate-buffered saline (PBS), $7 \mathrm{M}$-urea and $4 \mathrm{M}$-guanidine hydrochloride $(\mathrm{Gdn}-\mathrm{HCl})$. Proteoglycans from individual extracts were fractionated by DEAE-Sephacel chromatography and gel chromatography on Sephacryl S-400. The presence of three populations of large proteoglycans was demonstrated in all three extracts by composite agarose/polyacrylamide-gel electrophoresis (CAPAGE). The population corresponding to the fastest CAPAGE band of aggregating proteoglycans was shown to be extremely polydisperse, having $M_{\mathrm{r}}$ (as estimated by SDS/PAGE) decreasing continuously from more than 300000 to the size corresponding to 'free' hyaluronic acid-binding region (HABR) (about 70000). A rather polydisperse set of HABRcontaining fragments which spanned a broad range of sizes, and also differed in their keratan sulphate contents, was isolated from both $7 \mathrm{M}$-urea and $4 \mathrm{M}-\mathrm{Gdn}-\mathrm{HCl}$ extracts. PBS and $7 \mathrm{M}$-urea extracts, but not the $\mathrm{Gdn}-\mathrm{HCl}$ extract, further contained small proteoglycans, identified as fast-migrating bands on CAPAGE electrophoretograms. One of those small species was recognized with an antibody against the small proteoglycan PG II; the other two remain to be positively identified. However, the glycosaminoglycan of the small species which was present exclusively in the PBS extract was identified as keratan sulphate; this species may thus belong to the family of small keratan sulphate-containing proteolygans.
\end{abstract}

\section{INTRODUCTION}

Two main techniques have been used to demonstrate heterogeneity of large aggregating proteoglycans extracted from human articular cartilage. First, size fractionation by gel chromatography separates large proteoglycans into two populations [1-3], i.e. the large one chondroitin sulphate-rich and the smaller one keratan sulphate-rich. Secondly, the technique of electrophoresis in composite agarose/polyacrylamide gels (CAPAGE) separates large proteoglycans from human articular cartilage into three bands [1,4]; the reason for exactly this pattern of banding has not been clear. Finally, human articular cartilage has been reported to contain a relatively small fragment of the proteoglycan molecule which still retains the ability to bind to hyaluronic acid [5].

The other proteoglycans present in cartilage belong to the family consisting of two very similar species of small proteoglycans, which are designated PG I and PG II [6,7]; both of them are present in juvenile [8], but only one of them (PG II) is present in adult, human articular cartilage [9].

In total, several populations or fragments of large aggregating proteoglycans, together with one or two species of small proteoglycans, have been found in human articular cartilage by various authors; the list may not yet be final. We present here an attempt to make a systematic inventory of proteoglycans present in human articular cartilage, taking advantage of a selective extraction of different proteoglycans with solvents of increasing extraction efficiency.

\section{EXPERIMENTAL}

Tissues and materials

Human articular cartilage was obtained from femoral heads of two adults ( 29 and 87 years old) at the time of autopsy. In both cases autopsy was performed within $12 \mathrm{~h}$ of death, and the cartilage appeared macroscopically normal. Tissue was kept frozen at $-60^{\circ} \mathrm{C}$ until further processing.

Chemicals used were of analytical-reagent grade and obtained from standard commercial sources.

\section{Extraction of proteoglycans}

Full-depth cartilage slices were frozen in a known volume of distilled water and sectioned ( $20 \mu \mathrm{m}$ thick) on a freezing cryostat [1]. Care was taken to recover all resulting sections, including all water used to freeze the samples. The same volume of twiceconcentrated phosphate-buffered saline, pH 7.4 (PBS), including twice-concentrated proteinase inhibitors, was then added to the sample. The volume was then made up to $15 \mathrm{ml} / \mathrm{g}$ wet wt. of tissue, with the PBS containing proteinase inhibitors $(5 \mathrm{mM}$ phenymethanesulphonyl fluoride, $5 \mathrm{~mm}$-benzamidine, $5 \mathrm{~mm}-6$ aminohexanoic acid, $5 \mathrm{mM}-\mathrm{N}$-ethylmaleimide and $10 \mathrm{mM}$ EDTA). The tissue was extracted for $24 \mathrm{~h}$ at $4{ }^{\circ} \mathrm{C}$. The extract was separated from the residual tissue by centrifugation $\left(8^{\circ} \mathrm{C}\right.$, $50000 \mathrm{~g}, 60 \mathrm{~min}$ ), the pellet was washed with PBS, and the washing solution was added to the extract. The tissue was successively extracted with a solution of $7 \mathrm{M}$-urea/0.15 M$\mathrm{NaCl} / 50 \mathrm{~mm}$-Tris/HCl, $\mathrm{pH} 6.5$ (7 M-urea), containing proteinase inhibitors as above, under exactly the same conditions as above. The final extraction was with $4 \mathrm{M}$-guanidine hydrochloride/ $50 \mathrm{~mm}$-sodium acetate, $\mathrm{pH} 5.8(4 \mathrm{M}-\mathrm{Gdn}-\mathrm{HCl})$ (inhibitors and conditions as above).

\section{Papain digestion}

The residual tissue after a completed set of extractions was quantitatively transferred into dialysis tubing and extensively dialysed against distilled water. The tissue was then freeze-dried, weighed, and portions were solubilized with papain in $0.5 \mathrm{M}$ -

Abbreviations used: PG, proteoglycan; HABR, hyaluronic acid-binding region; CAPAGE, composite agarose/polyacrylamide-gel electrophoresis; $\mathrm{Gdn}-\mathrm{HCl}$, guanidine hydrochloride; $\mathrm{PBS}$, phosphate-buffered saline $\left(8 \mathrm{~g}\right.$ of $\mathrm{NaCl}, 1.15 \mathrm{~g}$ of $\mathrm{Na}_{2} \mathrm{HPO}_{4}$ and $0.2 \mathrm{~g}$ of $\mathrm{KH}_{2} \mathrm{PO}_{4}$ per litre, $\left.\mathrm{pH} 7.2\right)$. 
sodium acetate/20 mm-cysteine/5 mm-EDTA, $\mathrm{pH} 6.8$, at $60^{\circ} \mathrm{C}$, overnight. Glycosaminoglycan concentration in the papain digest was determined by using the dye-binding assay.

\section{Isolation of proteoglycans from tissue extracts by chromatography on DEAE-Sephacel}

Chromatography was performed on the DEAE-Sephacel column $(1.6 \mathrm{~cm} \times 16 \mathrm{~cm})$ equilibrated with $7 \mathrm{M}$-urea $/ 0.15 \mathrm{M}$ $\mathrm{NaCl} / 50 \mathrm{~mm}-\mathrm{Tris} / \mathrm{HCl}, \mathrm{pH}$ 6.5. The PBS extract was prepared for chromatography by adjusting it to $7 \mathrm{M}$ with respect to urea, by adding solid urea; the urea extract was directly loaded on the column. The Gdn- $\mathrm{HCl}$ extract was changed into $7 \mathrm{M}$-urea/0.15 M$\mathrm{NaCl} / 50 \mathrm{~mm}$-Tris/HCl, $\mathrm{pH} 6.5$, by dialysis. After the extract was loaded, the column was washed with $7 \mathrm{M}$-urea/0.15 M- $\mathrm{NaCl}$ until the $A_{280}$ in the eluate returned to zero, and was then eluted with a linear gradient of $0.15-1.0 \mathrm{M}-\mathrm{NaCl}$ in $7 \mathrm{M}$-urea at a flow rate of $10 \mathrm{ml} / \mathrm{h}$. The eluate was monitored for protein at $280 \mathrm{~nm}$; $5 \mathrm{ml}$ fractions were collected and assayed for glycosaminoglycan content by a dye-binding assay. Fractions were pooled, dialysed and freeze-dried. The pools separated by the chromatography on DEAE-Sephacel are designated in the text and Figures below by Roman numerals.

\section{Gel chromatography on Sephacryl S-400}

Chromatography on a Sephacryl S-400 column $(1.2 \mathrm{~cm} \times$ $150 \mathrm{~cm}$ ) was performed in $4 \mathrm{M}-\mathrm{Gdn}-\mathrm{HCl} / 50 \mathrm{~mm}$-sodium acetate, $\mathrm{pH}$ 5.8. Either the whole freeze-dried DEAE-Sephacelseparated pools (when weighing up to $10 \mathrm{mg}$ ) or portions of them weighing approx. $8 \mathrm{mg}$ were applied on the column. The column was eluted at a flow rate of $8 \mathrm{ml} / \mathrm{h}$. The eluate was monitored for protein at $280 \mathrm{~nm} ; 4 \mathrm{ml}$ fractions were collected and assayed for glycosaminoglycan content by a dye-binding assay. Fractions corresponding to individual peaks were pooled, dialysed and freeze-dried. The peaks separated by chromatography on Sephacryl S-400 are designed in the text and Figures below by Arabic numerals.

\section{Electrophoretic techniques}

SDS/PAGE on 4-20\%-polyacrylamide gradient gels was performed as described [10,11]. Gels were stained successively with Coomassie Blue and Alcian Blue [10]. The destained gels were dehydrated in concentrated solution of poly(ethylene glycol) 2000; shrunken gels were dried between two cellophan sheets.

CAPAGE was performed essentially as described by Carney et al. [4]. The running buffer, however, contained no urea, and gels were run in a tap-water-cooled electrophoresis apparatus. Gels were stained with $0.2 \%$ Toluidine Blue in $0.1 \mathrm{M}$-acetic acid, destained in $3 \%(\mathrm{v} / \mathrm{v})$ acetic acid, and dried on GelBond films. As the amount of sample loaded is critical for obtaining satisfactory resolution by CAPAGE, samples for electrophoretic analysis of proteoglycans were prepared as follows. Ethanol precipitates, or freeze-dried samples, were dissolved in distilled water and assayed for glycosaminoglycan content. Portions corresponding to $30 \mu \mathrm{g}$ of chondroitin sulphate were freezedried and dissolved again in $60 \mu \mathrm{l}$ of $60 \%$ (w/v) sucrose; $6 \mu \mathrm{l}$ portions of such samples were then loaded on agarose/ polyacrylamide gel. For SDS/PAGE, 1 vol. of a solution containing 4-times-concentrated sample buffer, $8 \%$ SDS and $2 \%$ mercaptoethanol was added to 3 vol. of the sample already dissolved in sucrose solution; samples were then heated at $100^{\circ} \mathrm{C}$ for $2 \mathrm{~min}$. The amount of the sample that was found optimum for staining in agarose gels with Toluidine Blue also gave satisfactory results when it was stained with Alcian Blue in polyacrylamide gels.

\section{Immunoblotting}

Transfer to nitrocellulose sheet was performed in $0.04 \mathrm{M}-$ Tris/glycine buffer, $\mathrm{pH} 8.3$, containing $20 \%$ (v/v) methanol. After the transfer, the nitrocellulose was blocked with $5 \%(\mathrm{w} / \mathrm{v})$ low-fat milk solution in PBS. Primary as well as secondary antisera were diluted in $2 \%$ milk solution in PBS. The following antibodies were used for immunodetection: rabbit anti-(pig cartilage PG II) antiserum [9], rabbit anti-[human hyaluronic acid-binding region (HABR)] antiserum, and the monoclonal antibody MZ15, which is specific for keratan sulphate [12]. All antibodies were kindly provided by Dr. T. E. Hardingham and Dr. M. T. Bayliss, Kennedy Institute of Rheumatology, London, U.K. The bound antibodies were localized with a peroxidaseconjugated second antibody.

\section{Determination of glycosaminoglycan concentration}

Two versions of a dye (1,9-Dimethyl Methylene Blue)-binding assay were used.The first version employed the reagent $(16 \mathrm{mg} / \mathrm{l})$ dissolved in sodium formate/formic acid exactly as described [13], and used $40 \mu \mathrm{l}$ samples plus $250 \mu \mathrm{l}$ of the dye solution. The linear range of this version was $5-50 \mu \mathrm{g}$ of chondroitin sulphate $/ \mathrm{ml}$. The less sensitive version employed the reagent (32 mg/l) dissolved in $0.04 \mathrm{M}$-glycine/0.04 $\mathrm{M}-\mathrm{NaCl}, \mathrm{pH} \mathrm{3.0,} \mathrm{and}$ used $10 \mu \mathrm{l}$ samples. The linear range of this version was $50-500 \mu \mathrm{g}$ of chondroitin sulphate $/ \mathrm{ml}$. Both assays were adapted for automated use with an e.l.i.s.a. reader. Calibration curves and blanks for every set of samples were always prepared in an appropriate solution (PBS, 7 M-urea, $4 \mathrm{M}-\mathrm{Gdn}-\mathrm{HCl}$ ).

\section{RESULTS}

Extraction of proteoglycans from the cartilage and isolation of proteoglycans from individual extracts by chromatography on DEAE-Sephacel

Slices $(20 \mu \mathrm{m})$ prepared from two differently aged (adult and very old) cartilage specimens were successively extracted with PBS, $7 \mathrm{M}$-urea $/ 0.15 \mathrm{M}-\mathrm{NaCl}$, and $4 \mathrm{M}-\mathrm{Gdn}-\mathrm{HCl}$, and residual tissues were finally solubilized with papain. Samples of individual extracts were assayed for sulphated glycosaminoglycans (Table 1).

The results of proteoglycan isolation are demonstrated only for the 29-year-old cartilage specimen from this stage onwards; differences that were found between the two differently aged tissues are discussed separately. A flow diagram summarizing the experimental protocol is shown in Fig. 1. Individual extracts were chromatographed on a column of DEAE-Sephacel in $7 \mathrm{M}$ urea (Fig. 2). Proteoglycans were eluted with a linear $\mathrm{NaCl}$ gradient as two or three rather broad and overlapping peaks.

Table 1. Glycosaminoglycan content in two specimens of human articular cartilage and their successive extracts

Glycosaminoglycan (GAG) content in each successive extract was determined by dye-binding asiay, with chondroitin sulphate (CS) as a standard. Total glycosaminoglycan content was calculated as the sum of amounts determined in individual extracts.

\begin{tabular}{|c|c|c|c|c|c|}
\hline \multirow[b]{2}{*}{ Age } & \multirow{2}{*}{$\begin{array}{l}\text { GAG total } \\
\text { (mg of CS/g wet wt. } \\
\text { of cartilage) }\end{array}$} & \multicolumn{4}{|c|}{$\begin{array}{l}\text { GAG in extract } \\
(\% \text { of total) }\end{array}$} \\
\hline & & PBS & Urea & $\mathrm{Gdn}-\mathrm{HCl}$ & Papain \\
\hline 29 years & 54.7 & 12.0 & 31.0 & 38.7 & 18.7 \\
\hline 87 years & 38.6 & 9.5 & 22.2 & 44.0 & 25.1 \\
\hline
\end{tabular}




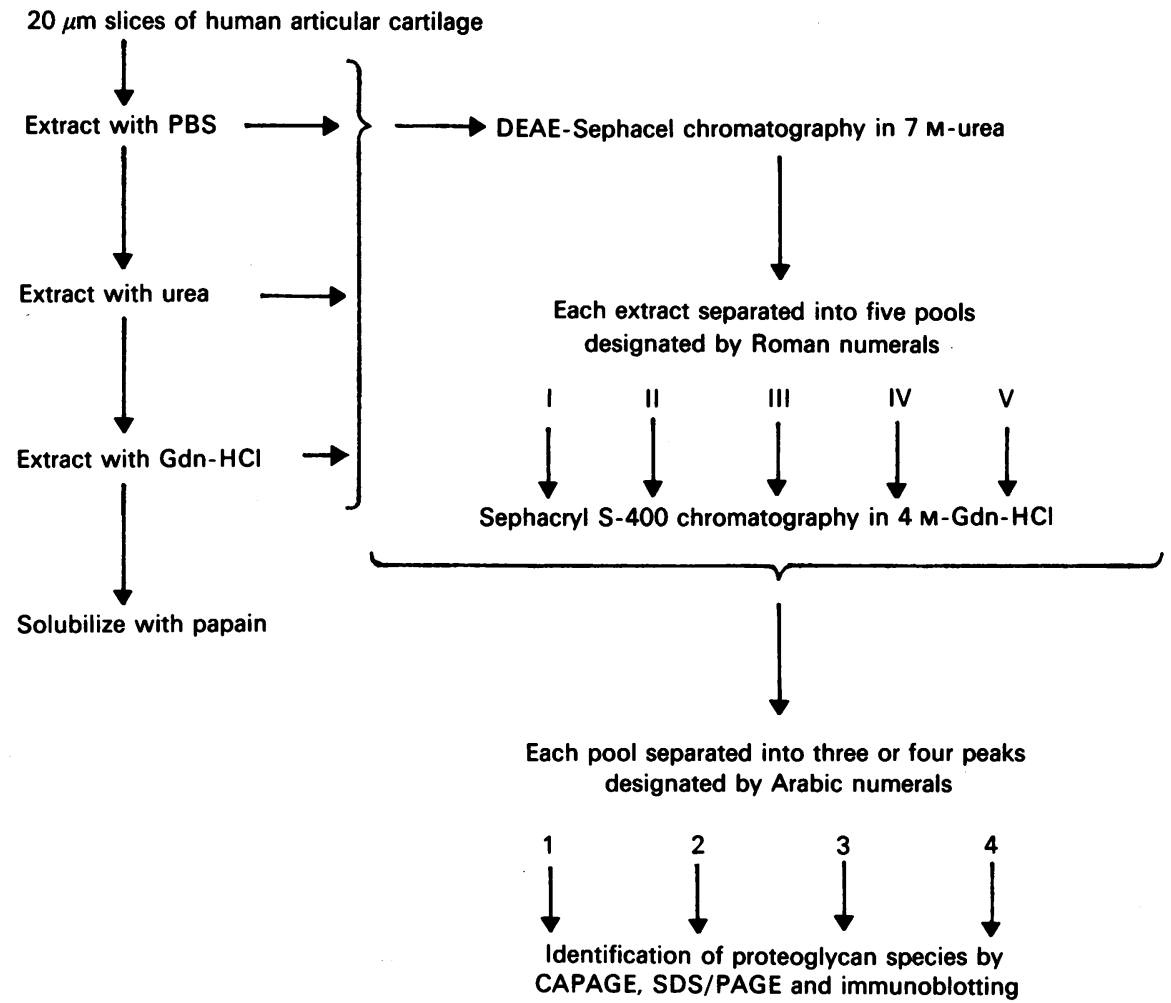

Fig. 1. Flow diagram outlining the experimental protocol used to isolate proteoglycans extracted from human articular cartilage
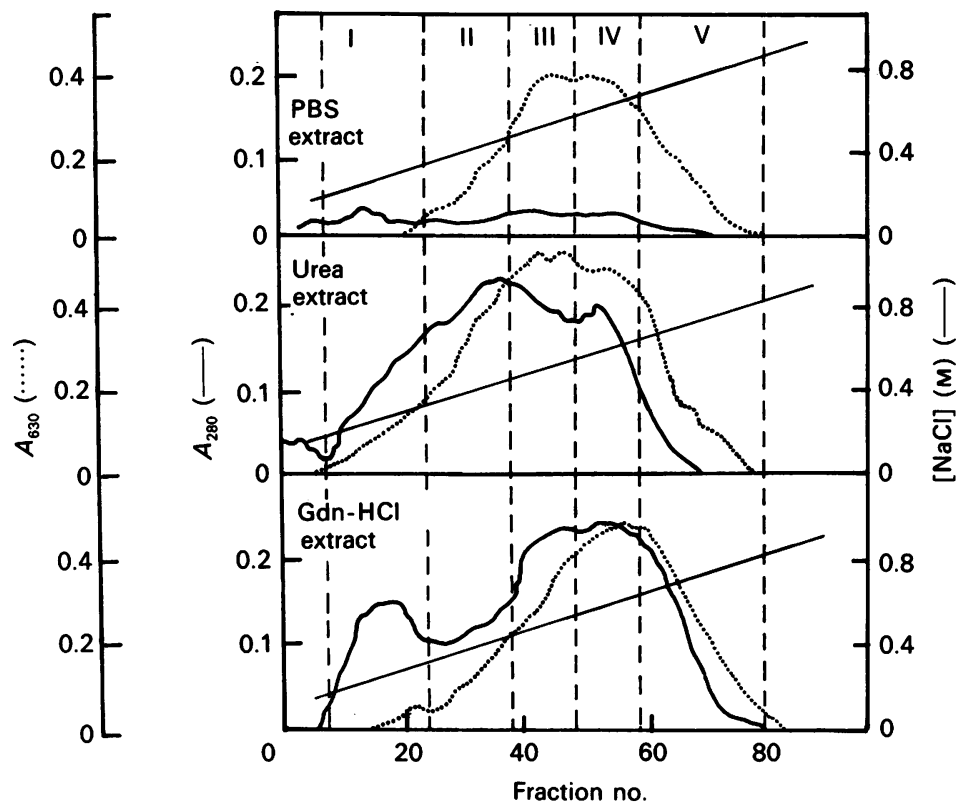

Fig. 2. Chromatography of proteoglycans successively extracted from human articular cartilage on DEAE-Sephacel in $7 \mathrm{M}$-urea

The eluate was monitored for protein $\left(A_{280}\right)$ and assayed for sulphated glycosaminoglycans $\left(A_{630}\right)$. Fractions were pooled as indicated (pools I-V).

CAPAGE analysis of every fourth fraction demonstrated that the three bands of large proteoglycans were eluted in a very broad range of the salt gradient for all three extracts (results not shown). Fractions were pooled as indicated in Fig. 2; individual DEAE-Sephacel-separated pools are designated by Roman numerals in Fig. 2 and in the text below. The yields of freeze-dried material, corresponding to individual pools, are summarized in Table 2.
Size fractionation of DEAE-Sephacel-separated pools by chromatography on Sephacryl S-400

Pools separated by DEAE-Sephacel chromatography were further fractionated by gel chromatography on Sephacryl S-400 (Fig. 3). This step separated proteoglycans into a maximum of four peaks which, however, were not equally represented in individual DEAE-Sephacel-separated pools. The $K_{\mathrm{av}}$. values of 
Table 2. Yields of freeze-dried material recovered in individual pools which were separated by DEAE-Sephacel chromatography

The total amount of wet cartilage extracted was $7.1 \mathrm{~g}$.

\begin{tabular}{|c|c|c|c|c|c|}
\hline \multirow{2}{*}{ Extract } & \multicolumn{5}{|c|}{ Yield (mg/g wet wt. of cartilage) } \\
\hline & I & II & III & IV & $\mathbf{V}$ \\
\hline PBS & 0.6 & 1.2 & 2.5 & 1.7 & 1.4 \\
\hline Urea & 2.3 & 4.7 & 5.0 & 4.5 & 2.5 \\
\hline Gdn-HCl & 1.4 & 1.4 & 1.5 & 2.9 & 7.8 \\
\hline
\end{tabular}

these peaks were $0,0.24,0.43$, and 0.58 ; they were designated peaks 1, 2, 3 and 4 respectively. Freeze-dried preparations recovered from pooled fractions which corresponded to individual peaks are designated in the text and in Figures below correspondingly by Arabic numerals. Portions of every second fraction from all gel chromatographies were precipitated with ethanol and analysed by CAPAGE and SDS/PAGE; the results are, however, illustrated below always on one representative electrophoretogram. It should be stressed here that the direct quantitative comparison between individual chromatographic profiles shown in Fig. 3 is not possible, owing to the differences

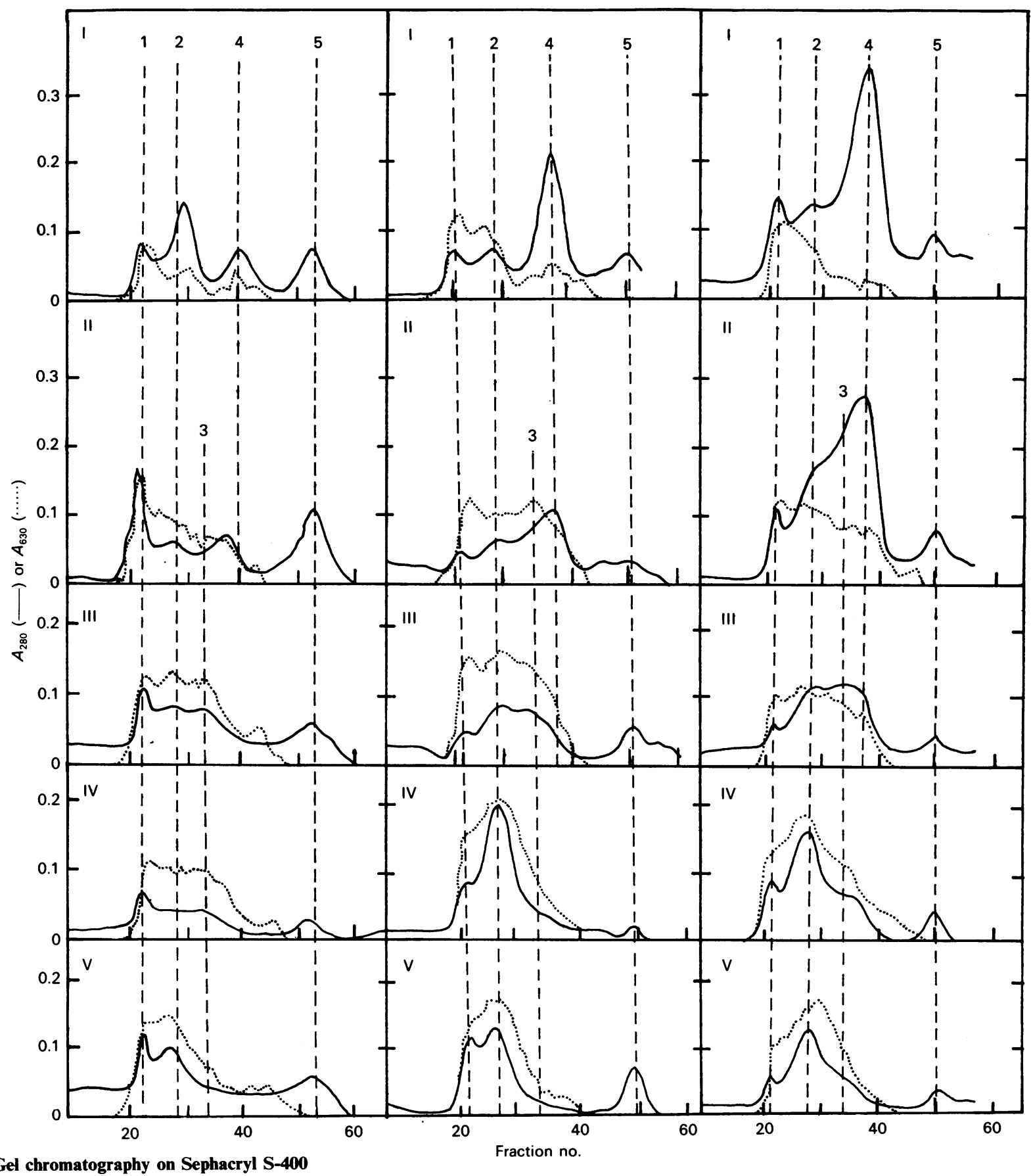

Fig. 3. Gel chromatography on Sephacryl S-400

The chromatographed samples represent portions of individual pools prepared by DEAE-Sephacel chromatography (as indicated by Roman numerals in the upper left-hand corners of individual chromatograms). The eluate was monitored for protein $\left(A_{280}\right)$ and assayed for sulphated glycosaminoglycans $\left(A_{630}\right)$. Broken vertical lines designated by Arabic numerals indicate the positions of peaks referred to the in the text. Peaks 1 and 5 corresponded to $V_{0}$ and $V_{\mathrm{t}}$ of the column respectively. 
in yields of material between individual DEAE-Sephacelseparated pools (Table 2). Similarly, all electrophoretograms demonstrated below indicate solely qualitative differences between proteoglycans eluted in chromatographic fractions analysed, as the amount loaded on individual electrophoretic tracks was always standardized.

\section{Identification of proteoglycans present in $\mathbf{G d n}-\mathbf{H C l}$ extract}

Proteoglycans present in the $\mathrm{Gdn}-\mathrm{HCl}$ extract were separated by Sephacryl S-400 chromatography into three major com-

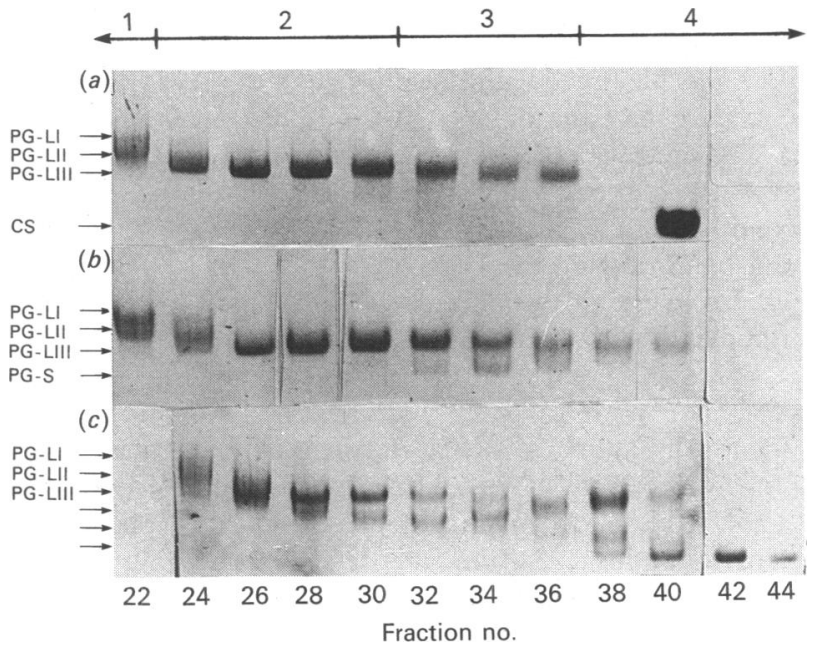

Fig. 4. CAPAGE of fractions separated by Sephacryl S-400 chromatography

Gel chromatographies shown are (cf. Fig. 3): (a) pool III of the $\mathrm{Gdn}-\mathrm{HCl}$ extract; $(b)$ pool II of the $7 \mathrm{M}$-urea extract; (c) pool III of the PBS extract. Arrows indicate proteoglycan bands according to their designation in the text. Track numbers refer to numbers of chromatographic fractions. Bars above electrophoretograms indicate pooled fractions (the corresponding chromatographic peaks are designated in Fig. 3 by the same numbers). Abbreviation: CS, chondroitin sulphate standard.

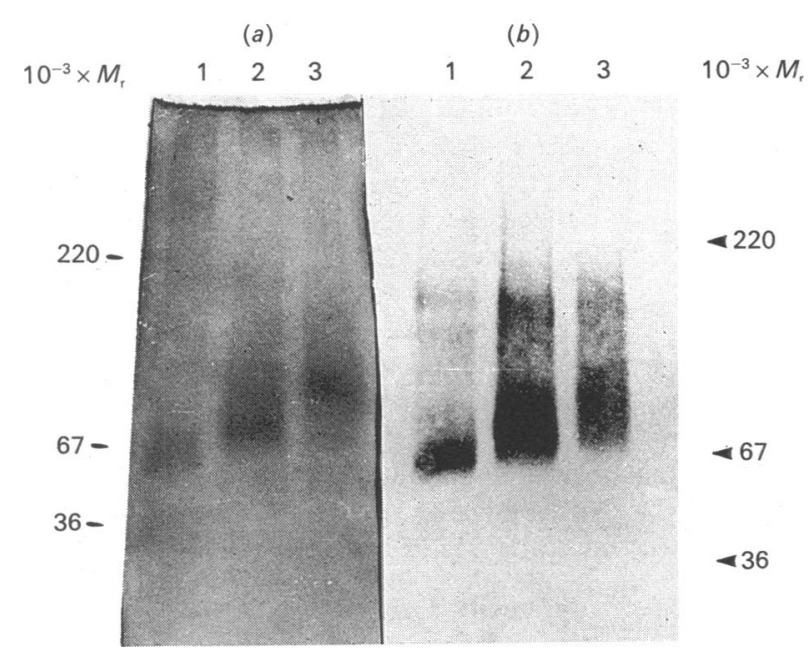

Fig. 5. SDS/PAGE and immunoblot of HABR-containing fragments isolated from the Gdn-HCl extract

(a) SDS/PAGE in 4-20\% gradient gel, stained successively with Coomassie Blue and Alcian Blue. (b) As (a), transferred by electroblotting to nitrocellulose and stained with anti-HABR antiserum as the primary antibody. Track 1, peak 4 of the Sephacryl S-400 chromatography of the DEAE-Sephacel-separated pool I; track 2, peak 4, pool II; track 3, peak 4, pool III. ponents (peaks 1, 2 and 4; cf. Fig. 3). Peaks 1 and 2, present in gel chromatograms of all pools, corresponded to individual bands of the characteristic CAPAGE triplet of bands, which represent different populations of large aggregating proteoglycans. Peak 1 corresponded to two slower-migrating CAPAGE bands (designated here as PG-LI and PG-LII); peak 2 corresponded to the fastest band of the triplet (designated here as PG-LIII) (Fig. 4). Pools I and II further contained a prominent protein peak, separated by Sephacryl S-400 chromatography (Fig. 3, peak 4). The material of peak 4 of pool I stained in polyacrylamide gels with Coomassie Blue and migrated on SDS/PAGE with $M_{\mathrm{r}}$ about 70000 ; when analysed by an immunoblotting, this material reacted strongly with the anti-HABR antibody. The corresponding materials (i.e. Sephacryl S-400-separated peaks 4) from pools II and III had somewhat higher $M_{\mathrm{r}}$; the increase in $M_{\mathrm{r}}$ with the pool number was detected on gels stained successively with Coomassie Blue and Alcian Blue, as on immunoblots stained with the anti-HABR antibody (Fig. 5). An increased $M_{\mathrm{r}}$ corresponded to more intensive reaction with the antibody MZ15

(a)

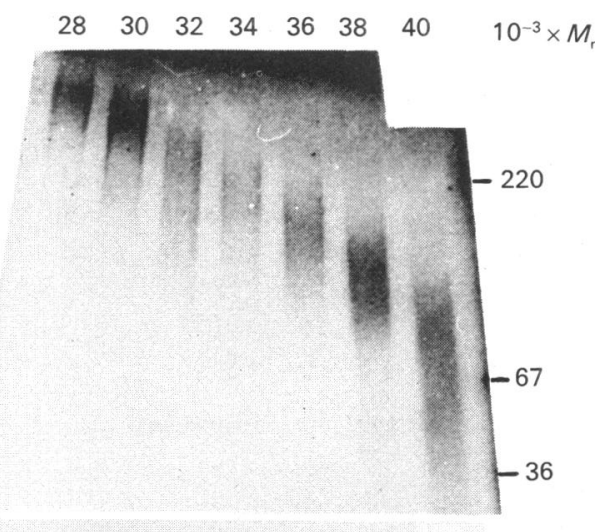

(b)

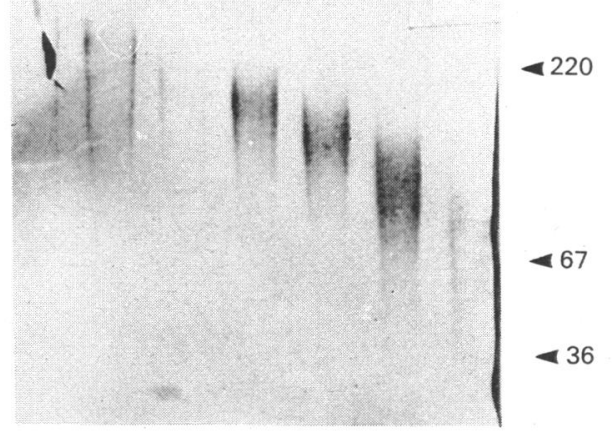

(c)

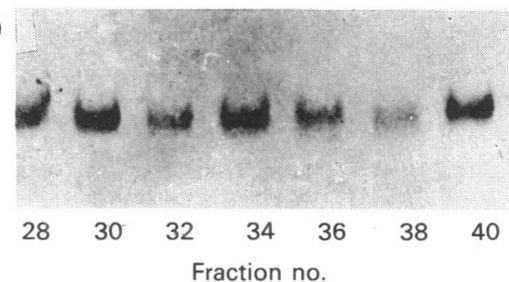

Fig. 6. SDS/PAGE and immunoblots of fractions separated by Sephacryl S-400 chromatography of pool III of the Gdn-HCl extract

(a) SDS/PAGE in 4-20\% gradient gel, stained successively with Coomassie Blue and Alcian Blue. (b) As (a), transferred by electroblotting to nitrocellulose and stained with anti-HABR antiserum as the primary antibody. (c) CAPAGE of the same chromatographic fractions, transferred by electroblotting to nitrocellulose and stained with anti-HABR antiserum as the primary antibody. Track numbers refer to the numbers assigned to the chromatographic fractions. 
(results not shown); in accordance, the material of higher $M_{\mathrm{r}}$ ceased to be stained with Coomassie Blue and started to be stained with Alcian Blue. The second band, of $M_{\mathrm{r}}$ about 150000 , was visible on anti-HABR-antibody-stained immunoblots, in addition to the main band of $M_{\mathrm{r}} 70000$ (Fig. 5). The $M_{\mathrm{r}}$ values of these two bands thus corresponded to values which were reported for 'free' G1 ( $N$-terminal hyaluronic acid-binding domain) and for the G1-G2 domain respectively [14].

There was a considerable polydispersity within the population of large proteoglycans represented by the CAPAGE band PGLIII. Proteoglycans containing the HABR epitope were detected in column fractions eluted with a $K_{\text {av. }}$ ranging from 0 to about 0.6 or even more; the $M_{\mathrm{r}}$ of corresponding proteoglycans (as estimated by SDS/PAGE) decreased continuously from molecules which did not enter the $4 \%$-polyacrylamide gel to about 70000 (Fig. 6). The HABR epitope (Fig. 6b), as well as keratan sulphate, which reacted with the MZ15 antibody (result not shown), were present in all these size fractions. The weaker immunostaining of material present in fractions 28-32 (Fig. 6b) was not caused by the lack of the epitope, but rather by a poor blotting of these relatively large proteoglycans on nitrocellulose. The material of peak 4 , consisting presumably mainly of 'free' HABR, thus appeared to represent an extremity of sizes which decreased to the size of this smallest fragment from the size of a native molecule of the large aggregating proteoglycan. Surprisingly, regardless of considerable differences in their hydrodynamic sizes, all these fragments migrated with essentially identical mobility on CAPAGE (Fig. $6 c$ ); the reason for this behaviour was not clear. The three populations of large aggregating proteoglycans (PG-LI, PG-LII, PG-LIII), and their small HABR-containing fragments, represented all proteoglycans that we have detected in $\mathrm{Gdn}-\mathrm{HCl}$ extracts.

\section{Identification of proteoglycans present in 7 M-urea extract}

Peaks 1 and 2, which corresponded to three populations of large proteoglycans, were evident in all Sephacryl S-400 chromatograms of individual pools (compare Figs. 3 and $4 b$ ). DEAE-

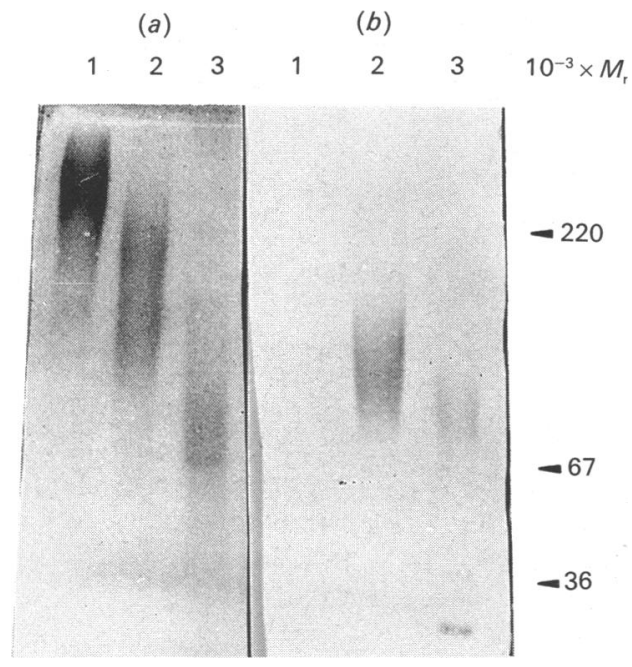

Fig. 7. SDS/PAGE and immunoblot of Sephacryl-S-400-chromatographyseparated peaks 2, 3 and 4 (pool II of the urea extract)

(a) SDS/PAGE in 4-20\% gradient gel, stained successively with Coomassie Blue and Alcian Blue. (b) As (a), transferred by electroblotting to nitrocellulose and stained with anti-(small PG II) antiserum as the primary antibody. Track 1 , peak 2 of the Sephacryl S-400 chromatography of the pool II; track 2, peak 3, pool II; track 3 , peak 4 , pool II.
Sephacel-separated pools I, II and III contained a component which was eluted from the Sephacryl S-400 column as peak 4 in pools I and II $\left(K_{\text {av. }}=0.56\right)$ and as peak 3 in pool III $\left(K_{\text {av. }}=\right.$ $0.42)$; similarly to the $\mathrm{Gdn}-\mathrm{HCl}$ extract, all analyses indicated that this material corresponded to small fragments of large aggregating proteoglycans bearing both HABR and keratan sulphate epitopes (results not shown).

Pools II and III further contained a small proteoglycan which was eluted from the Sephacryl S-400 column in the position of peak 3 and was identified as a distinct fast-migrating band on CAPAGE (band designated PG-S; fractions 32-36 in Fig. 4b). Pooled materials of peaks 2, 3 and 4 (which were separated by Sephacryl S-400 chromatography of pool II) were analysed by SDS/PAGE and by immunoblotting (Fig. 7). The small proteoglycan was identified with the anti-(small PG II) antibody which recognized the band of $M_{\mathrm{r}} 120000-150000$ in the peak 3 (Fig. $7 b$ ). The other bands separated by SDS/PAGE from peaks 2, 3 and 4 , i.e. a broad band of $M_{\mathrm{r}}$ about 300000 in peak 2, the second band of $M_{\mathrm{r}}$ equal to approx. 150000-200000 in peak 3, and a band ranging from $M_{\mathrm{r}} 70000$ to about 120000 in peak 4 (Fig. 7a), were all recognized by both anti-HABR and MZ15 antibodies.

\section{Identification of proteoglycans present in PBS extract}

Identically as in $\mathrm{Gdn}-\mathrm{HCl}$ and in urea extracts, the HABR epitope was present in the CAPAGE band which was designated PG-LIII (this band was again present in all Sephacryl S-400 chromatographies); again, all HABR-containing fragments migrated on CAPAGE with the same mobility (results not shown).

At least three distinct fast-migrating bands of small proteoglycans can be distinguished in Fig. 4(c), in positions indicated by unmarked arrows. They differed from each other in their electrophoretic mobilities which increased with decreasing hydrodynamic size (as indicated by the order of elution from the Sephacryl S-400 column). The first of these three bands (present in all DEAE-Sephacel-separated pools) was eluted from the Sephacryl S-400 column in fractions 28-34 and reached a peak in fraction $32\left(K_{\mathrm{av} .}=0.26\right)$. The second band (present in pools II-IV) was eluted in fractions $34-40$ (Sephacryl S-400 chromatography) and reached a peak in fraction $38\left(K_{\mathrm{av}} .0=0.38\right)$; it seems that this band was recognized by an antibody against small PG II, as this antibody selectively stained material present in fractions 34-38 (results not shown). When the same chromatographic fractions were analysed by SDS/PAGE and immunoblotting, the anti-PG II antibody recognized the band of identical $M_{\mathrm{r}}$ as in the urea extract, i.e. of about 120000 , in fractions 36 and 38 (results not shown).

The third fast-migrating band was identified by CAPAGE (in pools III, IV and V) in Sephacryl S-400-separated fractions 38-44 (Fig. 4c). This band corresponded to small peaks of sulphated glycosaminoglycans which were eluted from the Sephacryl S-400 column with $K_{\text {av. }}>0.6$ (Fig. 3). The material migrated with almost same mobility as the chondroitin sulphate standard on CAPAGE; however, it contained keratan sulphate, since it was recognized by MZ15 antibody (results not shown).

\section{Comparison of two differently aged samples}

To sum up, the differently aged samples which were examined simultaneously differed from each other in three respects. First, proteoglycans were extracted in a higher proportion by PBS and urea solution from the younger cartilage; also, the unextracted proportion which remained in the tissue was lower in that case (Table 1). Secondly, very old cartilage contained a relatively higher proportion of low- $M_{\mathrm{r}} \mathrm{HABR}$-containing fragments which were in higher proportion extracted already with 7 M-urea. 
DEAE-Sephacel-separated pools I and II, isolated from the $7 \mathrm{M}$ urea extract of 87-year-old cartilage, consisted on Sephacryl S400 chromatography almost solely of a single peak 4 , which was demonstrated to consist mainly of 'free' HABR domain. Thirdly, the relative proportion of small proteoglycans present in the very old cartilage was lower, compared with the adult tissue; on analysis of individual Sephacryl S- 400 chromatographies of 87-year-old cartilage by CAPAGE, the bands corresponding to small proteoglycans were visible only in the electrophoretograms of pools III of both PBS and $7 \mathrm{M}$-urea extracts.

\section{DISCUSSION}

Large proteoglycans and their HABR-containing fragments were present in all three successive extracts; both their proportions as well as their total contents in individual extracts increased with the extraction efficiency of the solvent. 'Free' HABR and the other small HABR-containing fragments can be supposed to be held in the tissue exclusively by virtue of their interaction with hyaluronic acid. However, their major part, as well as the major part of 'large' proteoglycan monomers, divided among the urea and $\mathrm{Gdn}-\mathrm{HCl}$ extracts. We hypothesized that the different extractibility of large proteoglycans might reflect the difference in stability of aggregates which either contain or lack the link protein. Whether these urea-extractable and $\mathrm{Gdn}-\mathrm{HCl}$-extractable populations of aggregating proteoglycans are indeed present in the tissue as 'aggregated-not-stabilized' and 'aggregated-linkprotein-stabilized' complexes with hyaluronic acid is at present an open question. On the other hand, the PBS-extracted large proteoglycans may not be present in the tissue in the form of aggregates. Since an immunoreactive HABR was present in at least part of the proteoglycans extracted with PBS, it remains to be determined whether this HABR is still functional.

We have demonstrated here the presence of fragments of large aggregating proteoglycans of extremely broad range of sizes already in the 29-year-old cartilage; the apparent $M_{\mathrm{r}}$ of the population designated as PG-LIII decreased from more than 300000 to the size of 'free' HABR domain. It has been reported that the content of the smaller keratan sulphate-rich proteoglycan in the human cartilage progressively increases with age [2], and that its proportion also increases through the cartilage depth [1]; both these findings, together with the polydisperity demonstrated here, support the hypothesis that this smaller population is generated from larger ones by partial degradation within the extracellular matrix.

The smallest HABR-containing fragments found in the Gdn$\mathrm{HCl}$ and urea extracts represented again a mixture, and included an almost 'pure' HABR and G1-G2 fragment. Purified HABR globe was shown previously to contain keratan sulphate [14-16]. The keratan sulphate content explains binding of all these fragments to the DEAE-Sephacel column and their staining with Alcian Blue; in the smallest fragments the keratan sulphate content was not, however, sufficient for satisfactory staining with Toluidine Blue in agarose gels.

Since all fragments of large proteoglycans containing the HABR globe migrated with essentially same mobility on CAPAGE, the CAPAGE technique could be employed to distinguish between large aggregating proteoglycans or their fragments and between the other 'small' proteoglycan species. These 'small' proteoglycans were extracted in significant amounts already with PBS, and 7 M-urea solution extracted them completely. Only one of three bands of small proteoglycans was positively identified, by means of immunoblotting, as PG II. The other two proteoglycans designated here as 'small' remain to be characterized. However, the keratan sulphate content indicated that the small species that migrated on CAPAGE as the fastest band might possibly be related to small keratan sulphatecontaining proteoglycans which have been isolated from cartilage recently $[17,18]$. Its exclusive presence in the PBS extract suggests that this proteoglycan is probably not firmly bound in the tissue.

The differences between two differently aged tissues which we report here were significant enough to become obvious from the comparison of essentially qualitative analyses. The main agedependent changes suggested here were the accumulation of 'free' HABR in the old tissue, and the decrease in the content of small proteoglycans with increasing age. Both these findings are congruent with results of Stanescu et al. [19], who identified small proteoglycans as a low-buoyant-density component of extracts of the young, but not of the old, human articular cartilage.

We thank Dr. T. E. Hardingham and Dr. M. T. Bayliss, who kindly gave us the antibodies used in this study, and Dr. P. Valouch, who dissected cartilage specimens. The excellent technical assistance of Blanka Kadicova is very much appreciated.

\section{REFERENCES}

1. Bayliss, M. T., Venn, M., Maroudas, A. \& Ali, S. Y. (1983) Biochem. J. 209, 387-400

2. Bayliss, M. T., Ridgway, G. D. \& Ali, S. Y. (1984) Biosci. Rep. 4, 827-833

3. Weber, C., Glant, T. T., Roughley, P. J. \& Poole, A. R. (1987) Biochem. J. 248, 735-740

4. Carney, S. L., Bayliss, M. T., Collier, J. M. \& Muir, H. (1986) Anal. Biochem. 156, 38-44

5. Roughley, P. J., White, R. J. \& Poole, A. R. (1985) Biochem. J. 231, 129-138

6. Fisher, L. W., Termine, J. D. \& Young, M. F. (1989) J. Biol. Chem. 264, 4571-4576

7. Neame, P. J., Choi, H. C. \& Rosenberg, L. C. (1989) J. Biol Chem. 264, 8653-8661

8. Roughley, P. J. \& White, R. J. (1989) Biochem. J. 262, 823-827

9. Sampaio, L. de O., Bayliss, M. T., Hardingham, T. E. \& Muir, H. (1988) Biochem. J. 254, 757-764

10. Fisher, L. W., Termine, J. D., Dejter, S. W., Whitson, S. W., Yanagishita, M., Kimura, J. H., Hascall, V. C., Kleinman, H. K., Hasell, J. R. \& Nilsson, B. (1983) J. Biol. Chem. 258, 6588-6594

11. Rosenberg, L. C., Choi, H. U., Tang, L.-H., Johnson, T. L., Pal, S., Webber, C., Reiner, A. \& Poole, A. R. (1985) J. Biol. Chem. 260, 6304-6313

12. Mehmet, H., Scudder, P., Tang, P. W., Hounsell, E. F., Caterson, B. \& Feizi, T. (1986) Eur. J. Biochem. 157, 385-391

13. Farndale, R. W., Sayers, C. A. \& Barrett, A. J. (1982) Connect. Tissue Res. 9, 247-248

14. Fosang, A. J. \& Hardingham, T. E. (1989) Biochem. J. 261, 801-809

15. Heinegård, D. \& Hascall, V. C. (1974) J. Biol. Chem. 249, 4250-4256

16. Bonnet, F., Dunham, D. G. \& Hardingham, T. E. (1985) Biochem. J. 228, 77-85

17. Plaas, A. H. K., Ison, A. L. \& Ackland, J. (1989) J. Biol. Chem. 264, 14447-14454

18. Oldberg, A., Antonsson, P., Lindbom, K. \& Heinegård, D. (1989) EMBO J. 8, 2601-2604

19. Stanescu, V., Chaminade, F. \& Muriel, M.-P. (1988) Connect. Tissue Res. 17, 239-252

Received 22 February 1990/24 July 1990; accepted 9 August 1990 